\title{
Foraging Wild Resources and Sustainable Economic
}

\section{Development}

\author{
Serge Svizzero ${ }^{*}$ \\ ${ }^{1}$ Université de La Réunion, Faculté de Droit et d'Economie, France \\ * Serge Svizzero, E-mail: Serge.svizzero@univ-reunion.fr
}

\begin{abstract}
As exemplified by the MDGs'adoption in 2000, it was recently thought that poverty alleviation, hunger reduction and environmental conservation should be tackle simultaneously. For that purpose, forests people had to intensively exploit and to commercialize the wild resources (NTFPs) they usually foraged from the nature for their self-consumption and for marginal trade. After a first wave of optimism, most studies have however concluded that such outcome was dubious, i.e., that foraging was not able to sustain economic development. We consider a theoretical framework and provide an economic analysis explaining such result. We identify four cases, according to two distinctions. First, foraged wild resources can be used either for self-consumption or traded on the market. Second, foraging can be either an exclusive mode of production or be combined with farming. However, an economic development based on the intensive exploitation of wild resources (through specialized foraging and trade) is not sustainable in the long-term. When considered as an economic system, the persistence of foraging wild resources is only consistent with self-consumption or with marginal trade. Intensive foraging dedicated to trade and market extension lead to the disappearance of sharing (or cooperation) between foragers; wild resources as well as most foragers are likely to get worse in such situation even though some counter-examples can be identified.
\end{abstract}

\section{Keywords}

foragers, farmers, self-consumption, NTFPs, communal sharing

\section{Introduction}

Foraging is usually defined as the search for wild food resources. Such behavior is ubiquitous among animals and humans. For animals, it is central because it affects their fitness and therefore their ability to survive and to reproduce. For the same reason foraging behavior is also important for humans. Such importance has been recently highlighted by the MDGs' adoption in 2000. Indeed, during the 1990s three major problems became global challenges in the agenda of economic development agencies: poverty alleviation, the struggle against hunger and famine, and ensuring environmental sustainability. The first two challenges were gathered in the first MDG-eradicate extreme poverty and hunger-while the third challenge was presented as the seventh MDG. In addition, it appeared that 
these three challenges were-under some circumstances - interdependent and therefore should be tackle simultaneously (Note 1). Indeed, in developing countries-especially those located in the tropical region - some of the poor people were living in the forests or in areas very close to the forests. Moreover, these "forest peoples" included foraging among their economic activities, and often it was even their main economic activity. Although they were poor, without cash income and capital, they had Local Ecological Knowledge (LEK) from which they were able to forage Non-Timber Forest Products (NTFPs (Note 2)) in order to get their subsistence. It was thus believed that if these forest peoples were able to exploit more intensively and to commercialize the NTFPs they foraged, then a win-win situation will emerge (Peters et al., 1989). On the one hand, these foragers would have a higher (and cash) income from which they might eradicate hunger and start an accumulation process in order to sustain their economic development. On the other hand, because they have LEK and they get their resources by foraging NTFPs, they would take care of the forests, i.e., they would actively contribute to biodiversity conservation and environmental sustainability. While such view has led to optimism in the $1990 \mathrm{~s}$ (Stiles, 1994; Delang, 2006a, 2006b), it has become progressively obvious that such view was dubious. Optimism was followed by skepticism and subsequently by pessimism, i.e., it became obvious that it was not possible to establish a sustainable economic development on a economic system mainly based on foraging (Shackelton et al., 2008; Sills et al., 2011; Stanley et al., 2012). For instance in a recent review of economic and ecological literature, Stanley et al. (2012, p. 1) conclude that "Roughly 75\% of studies demonstrated that gatherers earned more than USD\$2 PPP/day (the international absolute poverty line) or more than a local wage. These positive results do not, however, demonstrate that gathering reduces long-term poverty because forest dependence, and likely tenure security, remains low among these populations".

It is the aim of this paper to provide an economic analysis in a theoretical framework explaining why foraging - or more generally any economic system based mainly on extraction of resources from the wild - cannot lead to a sustained economic development. We therefore focus on the impact of intensified foraging and commercialization of wild resources on economic development. Even though it is of greatest importance, we do not deal in this paper with the impact of such strategy on biodiversity conservation (Note 3).

For human, while foraging is considered as crucial at the individual level, its role at the social level seems of less importance. Indeed, in an economic perspective, foraging is only associated with survival or self-reproduction rather than with capital accumulation and economic growth. Therefore, and by comparison for instance with the production of food resources, foraging cannot lead to a sustainable economic development. Yet, when we consider material resources—including food resources—humans may satisfy their needs according to two different economic systems, extraction or production. In the former, human use various inputs (land, labor, capital, human capital) to extract material resources from the wild. In other words, the resources are produced by the nature, extracted, processed and consumed by humans. In the latter, humans also use inputs in order to produce material resources, e.g., 
to produce food resources through agro-pastoralist processes. While extraction and production are potentially two competing economic systems, economic development is the outcome of only an economy of production.

An historical situation from the far past is useful because it illustrates why foraging cannot sustain economic development and therefore helps to explain the recent failure of foraging policies fostered by the MDGs adoption. It is associated with the pre-Neolithic period, i.e., before the introduction of food production which arose from 10.000 B.C. (Svizzero \& Tisdell, 2014); let us recall its main features (Kelly, 1995). During the Palaeolithic and the Mesolithic periods - and thus during 98\% of human life on earth-foraging was the only method available to get food resources. Foragers, called Hunter-Gatherers (HG), were roaming almost permanently to get their subsistence. Economic surplus, i.e., the excess of extraction over consumption, was extremely rare. When it existed, it was-according to Malthus's law_immediately used to increase the population level. The latter increased very slightly (Renfrew \& Bahn, 2012, p. 456) and HG remained structured as "bands", each band including few dozen of people linked by kinship. It is only with the introduction of agriculture and the so-called "Neolithic revolution" (Childe, 1936) that larger and more complex social organization appeared, such as tribes, chiefdoms and states (Service, 1975). It is also with the Neolithic revolution-and thereby under an economic system based on production - that the economic surplus grew large leading to the diversification of economic activities (pottery, metalworking, weaving...), the emergence of social and economic inequalities, and the introduction of features which characterized civilization (such as writings, government). Under the extraction economic system, HG remained locked at an early stage of economic development, considered by some authors as primitive or even savagery (Morgan, 1877).

The paper is organized as follows. Section 2 provides the definition of wild resources as well as some of their uses and the various goals of foragers. In section 3 we present a typology of what people do on the supply-side of the market (forager/farmer) as well as on the demand side (self consumption/trade). Section 4 is about what happens when foragers get specialized in order to trade their production on the market. Sharing, a central feature of foragers' societies, and the conditions of its breakdown, is presented in Section 5. Section 6 analyses what happens when foragers-farmers get specialized in order to trade on the market what they harvest from the wild. Section 7 concludes.

\section{Wild Resources: Extraction and Consumption}

The number and variety of resources that can be considered as "wild" is very large. It contains plants (trees, fruits, nuts, weeds, fungi, roots, tuber, lichen...), terrestrial animals (Note 4) (mammals, birds, insects...), aquatic animals (fish, mammals, shellfish...), minerals (stones, precious stones, sand, coal, salt...) and water. We introduce a restriction in the sequel about the scope of wild resources; indeed we will consider only renewable wild resources (minerals are therefore excluded of the scope of our analysis). Although there is no widely accepted definition of what NTFPs are precisely (Ahenkan \& Boon, 2011), it is clear that since they include all botanicals and other natural products extracted from 
the forest other than timber, NTFPs are also included in the previous list of wild resources.

\subsection{Foraging, or How the Resources are Extracted from the Wild?}

Foraging has been and still is ubiquitous in human life. At any age of their life, in any circumstances, people are foraging, i.e., they are searching for material or immaterial resources which could be provided by the nature or produced by man. For instance, and concerning immaterial resources or information, web-users surf the Internet for desired data, and traders on the financial market face the traditional foraging trade-off (Saavedra et al., 2013). Similarly, any consumer evolving in a shopping center is foraging produced material resources. However and for simplicity, we restrict our analysis to the foraging of material resources provided by the nature, i.e., to wild resources (as defined previously). Even though they are labeled differently, such as "wild resources", "forest products", or "NTFPs", they all have in common the fact that they are foraged or extracted - and not produced-from the nature. For this purpose, foragers use various techniques such as hunting, fishing, fowling, gathering, collecting (...). As for any production process, the techniques used by foragers require also inputs such as land (or water: sea, river, lake, marshes...), labor, capital and knowledge (human capital, called LEK). The analysis of the foraging process has been widely influenced by the study of animal foraging behavior. In the latter, a central result is provided by the marginal valuation theorem (Charnov, 1976). The Marginal Value Theorem is an optimality model that describes the strategy that maximizes gain per unit of time in systems where resources, and thus rate of returns, decrease with time. It is at the centre of optimal foraging theory which was applied initially to animal behavior (Pyke et al., 1977) and subsequently to human foraging behavior in what has been called "Human Behavioral Ecology" (denoted by HBE; Winterhalder \& Kennett, 2006). In HBE, any human forager is assumed to behave as an animal does. He may encounter two different situations. In the first one, various resources are distributed in a given patch and thus the forager has to maximize the difference between the energy (when it is about food resources) or income provided by collected resources and the energy (or income) spent as a result of movement from one resource to another. When such situation is about food resources, it leads to the optimal diet model (or the diet breath model). In the second situation, one resource is unevenly distributed among various patches. The same logic as in the first case applies, but costs are associated now to movement from one patch to another. This leads to the patch choice model (or the optimal location model).

\subsection{What the Foraged Wild Resources Are Used for?}

Whereas wild resources that can be foraged are numerous, it should also be noted that they can be used for various and numerous purposes (Svizzero, 2016). The common belief about their use is that they ensure subsistence. This can be true but in addition to provide food resources-for humans and also for animals, e.g., fodder - foraged wild resources may also satisfy other basic human needs. Among the latter are the foraging of wild resources for medicinal purpose, in order to build shelters, to make clothes, to get fuel (...). Beyond the previous basic needs, which correspond to a biological goal, wild resources may also be used for other purposes. Indeed they may have cultural, religious or social 
significance, e.g., for recreation (Note 5), and even ecological significance, i.e., when people wish to "commune" with nature as for instance most urban foragers do (McLain et al., 2014). There is thus a second goal associated with foraging, which can be defined as a socio-cultural goal.

In addition to the previous query, "what are they used for", many other features of wild resources, about their uses, are also important. For instance, how these resources are used? Should they be consumed on the spot or could they be stored? Once they are collected, could they be consumed directly as any final good or should they be first processed as any raw material? Among all the possible questions, one is of great importance for our purpose and is the following: who are consuming these wild resources? There can be two (non exclusive) answers. Either people who forage also consume the resources they extract: this is self-consumption, i.e., a situation in which people do not have reliance on the market to ensure their subsistence. Or people who forage sell on the market the resources they extract. They may sell on the market part of these resources (and then resources are used within the household and the market) or all the foraged resources (and then there is no self-consumption). Whatever the option they choose, their livelihood are dependent (completely or partially) on what will be the market equilibrium (i.e., the prevailing prices and the quantities exchanged). This defines the third goal of foraging, i.e., an economic goal. Another aspect of NTFPs stressed by Chambers (1987) is that in some developing countries they act as an economic security blanket to be drawn on in times of drought or economic hardship.

\section{Foragers/Farmers and Self-Consumption/Market Reliance: A Typology}

We have previously highlighted that the economic goal of foraging is associated with a first distinction between self-consumption and consumption from the market. It can be enriched by the introduction of a second distinction, i.e., whether people who extract wild resources are "pure" foragers or not. In the former case, they only forage, i.e., they do not produce any good. In the latter case, they forage and they also produce material resources; when for instance the latter include food resources, then people are called "foragers-farmers". It should however be noted that, as recall and detailed by Pryor (2004), the frontier between foraging and farming is often blurred. Indeed, many HG exhibited some types of proto-agriculture. For instance, while they are commonly considered as HG, Australian Aborigines were using "fire-stick agriculture" in order to favor some plants by clearing the others or to attract wild game in freshly cleared meadows.

We thus have two distinctions concerning wild resources: the first one is about the demand side, i.e., who are the users or consumers? The second one is about the supply side, i.e., who are the providers or producers? By combining these two distinctions, we get a typology of four distinct cases. 


\subsection{Case 1: "Pure Foragers"}

In the first case, people are "pure foragers", they self-consume what they extract. This is what we usually have in mind when we talk about hunter-gatherers, and more precisely about pre-Neolithic HG. Indeed, the latter were obviously not producers of food resources because agriculture was invented later. Even if there were some exchanges of goods between bands of HG, they were restricted to luxury items or "exotic" such as precious stones used for ornaments. In other words, market were not well developed during the prehistoric period. This was due to physical as well as economical constraints: resources extraction by HG provided low-level or even no surplus, transportation was not easy and insecure, medium of exchange were often absent, most HG were nomads and therefore there was no marketplace where supply and demand could be met (...). Today, instances of case 1 must be very rare.

\subsection{Case 2: "Foragers-Farmers"}

In the second case, people can be defined as "foragers-farmers", i.e., they gather wild resources, including food resources, and they also produce food resources through an agro-pastoralist process. However, and whatever the way they get their (extracted or produced) resources, the latter are totally or mainly used for self-consumption. In other words, the reliance on the market, in order to sell or to buy, is very limited. Potentially such situation may only exist once agriculture has been invented, i.e., at any period from the Neolithic period onwards. However it was more likely to occur when the productivity gap between the two economic systems, extraction and production, was low (Weisdorf, 2005, p. 583). This may correspond either to the early ages of agriculture or to region which are marginal for agricultural production (e.g., due to low soil fertility, water shortages, or harsh climate conditions). Such situation has been defined as "low-level food production" by Smith (2001); it is identified as a $30-50 \%$ dependence on domesticated plants and animals, the remaining food being derived from hunting and gathering. In fact, a variety of subsistence economies, extant, historic, and prehistoric, drew upon both elements of hunter-gatherer and agricultural modes of production (Svizzero \& Tisdell, 2015). These are difficult to characterize in existing terminologies except as 'mixed' economies (Winterhalder \& Kennett, 2006, pp. 2-4). Compared to case 1, case 2 seems still to be not that uncommon. The Mikea (living in the southwest of Madagascar) is an example of case 2 (Stiles, 1998).

\subsection{Case 3: "Foragers-Traders"}

As the second case is, the third case is also an extension of the first case but now we consider "foragers-traders". In this situation, foragers do not produce any goods, such as food resources or anything else, but they sell on the market a significant part of what they collect from the wild. Potentially, this may occur in a world in which only foragers are living; however it is more likely to occur in a world where agriculture also exists (and other economic activities based on production as well). Although it is not restricted to this continent, many examples of foragers-traders have existed and are still present in Asia (Morrison, 2005) and elsewhere also (Svizzero \& Tisdell, 2015). For Asia, numerous and various examples illustrating what is sold (or was sold in the past) by "foragers-traders" are provided by Morrison (2005, p. 285): rattans, precious woods (scented woods, camphor, 
sandalwood, teak...), ores (tin), bird's nests, sea slugs, bird of paradise feathers, hunted animals, honey, medicinal plants, spices (pepper, cardamom, ginger, clove, nutmeg...). For all continents, examples of foragers bands or tribes illustrating case 3 are for instance provided in Stiles (2001, pp. 45-46), and are presented in an historical perspective. This author also distinguishes sporadic exchange from regular and intensified exchange.

\subsection{Case 4: "Foragers-Farmers-Traders"}

The fourth case is complex and also wide-spread (Bharucha \& Pretty, 2010). Indeed, people must be considered as "foragers-farmers-traders". This means that they do foraging as well as farming, that they self-consume part of what they extract and/or produce and sell the remaining part on the market. This case is complex because there exists an infinite number of possible combinations of foraging/farming and of self-consumption/market-consumption. Indeed, the degree of dependence on NFTPs varies and their use of forests seem to vary. Moreover, many handcraft and artwork products sell to tourists by forest people all over the world are made from forest products (carved wood, shells...). Case 4 is widespread because it encompasses diverse groups of people, such as what are still considered as HG (e.g., Kung, Mbuti or Hadza in Africa, Pwo Karen or Mlabri in Thailand, Ache in Paraguay...), but also forest people, many agricultural communities (especially in developing countries) and, to some extent, urban foragers.

3.5 The Typology

Table 1. A Typology Based on Demand and Supply Sides Economic Activities

\begin{tabular}{lll}
\hline & Self-Consumption & Trade on the Market \\
\hline Only Foraging & Case 1 & Case 3 \\
Foraging and Farming & Case 2 & Case 4 \\
\hline
\end{tabular}

In the sequel, we will study two transitions:

From case 1 to case 3 (in section 4 )

From case 2 to case 4 (in section 6 )

Both transitions consider what happen when people decide to intensively exploit and commercialize wild resources. Please note that the transitions from case 1 to case 4 , and from case 2 to case 3 are not relevant.

The transition from case 1 to case 2 is not studied since it does not consider trade on the market, it is out of the scope of the present paper. For reasons explaining why such transition from foraging to farming is either effective, delayed, or impossible, see for instance Svizzero and Tisdell (2015).

The transition from case 3 to case 4 is not studied because it considers that commercialization of wild resources is already ubiquitous, whatever is the method (foraging or farming) used to get traded resources. 


\section{From Unspecialized Foraging and Self-Consumption to Specialized Foraging and Trade (Case}

\section{1 to Case 3)}

In this section we consider a simplified economy in which only foraging or resources extraction is possible. Initially all people self-consume the resources they extract from the wild (this is case 1, i.e., "pure foragers"). Afterward, they remain foragers but they are now able to self-consume and also to trade on the market the resources they extract (this is case 3, i.e., "foragers-traders"). Such evolution (from case 1 to case 3 ) rises three important questions. Before we turn to each of these three questions, let us first consider a necessary condition for foraging to exist, whatever foraged wild resources are used for.

\subsection{The Land Tenure System and Access to Wild Resources}

Even though it seems tautological, in order to forage, it is necessary on the supply-side that any forager be able to have access to the wild resources. This raises the problem of land tenure which is crucial for any economic development based on foraging. The major threat to the NFTPs used by tribals is the transformation of forested areas by non-tribals. This includes the conversion of forested land to agriculture (Swanson, 1994), e.g., the plantation of monoculture forests. Even though the access to wild resources is "warranted", the contribution of access of NFTPs to reducing poverty remains contentious. What is clear is that if some groups were denied access to such resources, then they would suffer increased poverty. This does not however mean that access to these resources and their possible scale is capable of raising the incomes of villagers that use these resources to any significant extent.

\subsection{Division of Labor, Surplus and Trade}

The first important question is why foragers decide to trade on the market (rather than self-consuming all the resources they forage)? If they decide to trade it is because some foragers have a comparative advantage in the extraction of some or at least one wild resources while other foragers have also a comparative advantage in the extraction of some other or at least one another wild resources. In other words, all foragers derived a surplus from their activities but for different resources. Therefore, they may trade each other their surplus throughout the market and thus they all improve their welfare. This is Ricardo's (1817) theorem of comparative advantage (Note 6) related to international trade which is here simply applied to different groups or bands of foragers rather than to different nations.

The second important question is what is necessary in order to trade foraged resources on the market? In addition to the previous condition, it is also necessary that any forager be able to have a surplus. This can occur naturally if, for instance, a wild resource is particularly abundant in some area and is also easy to collect, to transport and to store (e.g., wild cereals, acorns, nuts, shellfish, bamboos, rattan...). More generally, such surplus may be the result of specialized foraging. This is the application of Adam Smith's (1776) conclusion about the division of labor. Indeed, there can be a division of labor between foragers belonging to the same band or between different bands of foragers. Such division of labor may be the result of various influences such as the relative abundance of resources in different territories, the different abilities among foragers, institutions or the relative level of development. As demonstrated 
by Ruiz-Pérez et al. (2004) in a detailed analysis based on 61 cases (Note 7), the division of labor, i.e., the specialization of forest peoples, is mainly driven by markets. Under such division of labor, foragers become specialized; this specialization leads to an increase of their productivity (through learning by doing) and therefore to an increase of the surplus they extract. On the demand-side, the trade on the market of foraged resources requires several conditions. Foragers must have a physical access to the market, i.e., transportation costs (especially for remote foragers) must not be too excessive. Moreover, the resources they sell must be desired at any income level and their demand must be an increasing function of consumers' income. In other words, wild resources must be normal goods rather than inferior goods.

\subsection{Wild Resources: From Intensive Exploitation to Extinction}

The third important question is what are the consequences on the development of the foraging economy when foragers implement a more intensive and specialized extraction of wild resources? Even though the conclusions are the same, we may distinguish the consequences, on the one hand, on the economic profitability of foraging activities and, on the other hand, on the socio-economic organization of foragers. We first deal with the former consequences while the latter are presented in the next section (section 5).

When foragers decide to get specialized, this has two opposite effects on the productivity in foraging. On the one hand - and as highlighted previously - their productivity is improved due to learning by doing. On the other hand, excessive extraction-i.e., extraction done at any rate beyond the natural rate of growth of the wild resource considered-leads to a decrease of the stock of the resource; thus foraging exhibits diminishing returns. If excessive extraction is persistent, it may even lead to the extinction of the resource, as exemplified by Smith (1975) for the prehistoric megafauna extinction explained through the overkill hypothesis (Note 8). While the two effects on foragers' productivity are opposite, it is likely that the productivity will decrease continuously in the long-term. This is so because specialized foraging fosters excessive extraction. Indeed, competition among specialized foragers, i.e., the quest of continuous larger amount of surplus is supported by the increased level of the market demand and is also facilitated by the land tenure system of foraging economies, namely by common property regimes (Weisdorf, 2005, pp. 573-574; North \& Thomas, 1977). We reach here the following conclusion: specialized foraging does not lead to sustainable economic development because foragers' productivity is decreasing with the passage of time. Therefore, two cases are possible. First, after a while, forager's productivity is so low that specialization becomes less profitable than foraging without specialization. This leads foragers to give up their commitment with the market and to reverse to the initial situation, i.e., to forage only for their self-consumption. Second, even if it decreases continuously, the productivity provided by specialized foraging remains higher than in other activities. Excessive extraction is thus maintains until the stock of the concerned wild resource be completely depleted.

One may however object to the previous conclusion that some wild resources (e.g., bamboos) are very 
abundant whatever the amount extracted is, and therefore there is no reason that foragers' productivity decreases, i.e., such specialized foraging seems sustainable. In fact it is not, in any cases. This can be demonstrate as follows, through the analysis of the market demand of such wild resource. Two cases are possible. First, since the resource is abundant, we may assume that its supply remains always higher than the demand, and the latter may even saturate. Therefore the price of the resource remains very low and there is no obvious reason (except if they are constrained to) explaining why some foragers have decided to specialize in the extraction of this resource. Second, if the demand is not saturated (i.e., it is continuously increasing when the price tends to zero), despite the abundance of the resource, this means that the latter is very valuable for market's stakeholders. This will foster effort in order either to domesticate and then to produce this resource, or to produce goods which are close substitutes of the wild resource (e.g., most—if not all—products made from bamboos have substitutes goods made from plastic or metal). In both situations, the demand of the wild resource will decrease, as well as the profitability associated with its extraction.

We have demonstrated that an economic system based on specialized foraging and market-consumption cannot lead to a sustainable economic development. In other words, an economic system with foragers-traders is possible only if trade remains marginal, i.e., if the traded wild resources correspond to a fortuitous surplus. Such situation is illustrated by what are called "complex HG" societies (Price \& Brown, 1985; Keeley, 1988; Sassaman, 2004). Indeed, these "pure foragers" were labeled as complex HG (Note 9) because they were exploiting abundant and valuable resources (Note 10) and therefore were able to extract a significant surplus. However, most of this surplus was used for self-consumption (and therefore, according to Malthus' law, it has supported population growth of these societies) and only a marginal part of it was traded between bands of complex HG. Despite the existence of such surplus, any of these complex HG societies has not been able to trigger a significant economic development such as the one which has later been caused by the introduction of agriculture.

\section{Communal Sharing in Foraging Societies: A Central Feature and Its Breakdown due to Market Extension}

According to most (if not all) approaches of foraging, foragers' decision require a fine balance between the exploitation of their rewards and the exploration of different options (i.e., switching to another resource or to another patch). This balance is commonly referred to as the exploitation-exploration trade-off (Cohen et al., 2007; Berger-Tal et al., 2014; Mehlorn et al., 2015).

\subsection{Sharing, the Central Socio-Economic Feature of Foragers' Society}

It is also widely agreed in the academic literature that there is no optimal solution to the exploitation-exploration trade-off. However the latter is influenced by individual, social and environmental factors (Mehlorn et al., 2015). Among these influences, the social factor is of great importance for our purpose. Indeed, while foraging is asocial (or solitary foraging)-i.e., without competition or coordination with con-specifics-for many animals, it is not for all animals and 
especially for human. In fact socially foraging species (e.g., animals such as ants, felids...) minimize the exploitation-exploration trade-off by having individuals specialized in either exploitation or exploration and working together (Giraldeau \& Caraco, 2000). This is especially true for human, i.e., the analysis of human foraging behavior should be systematically done from a social foraging point of view. Indeed, human foraging is social and, in addition, it also includes a unique element (compared to what non-human animals do), the creation of resource pooling systems (Delton \& Robertson, 2012). In this type of social foraging, people contribute when they have excess resources and receive some provisions when in need. The latter may occur either because foraging is very risky - by nature it provides returns featured by high variance-or because injury and illness can prevent a person of foraging for extended periods. Resources pooling prevents of shortfalls, i.e., ensures risk reduction. It is also called "sharing" and is a feature of any group of foragers. For instance, sharing was a central feature of hunter-gatherers societies of the far past (e.g., during the pre-Neolithic period). While these pre-Neolithic HG were "pure foragers" (because agriculture has been introduced later), sharing is remained the central feature of "modern HG" societies, even though foraging was not for them the only method they use to get food resources (Lee, 2004; Lee \& Daly, 2004).

\subsection{Foraging, a Cooperative Economic Strategy}

From a microeconomic point of view, the extraction of wild resources can be compared to the production of goods. Indeed, both techniques can be simply compared throughout their respective productivity, e.g., the marginal productivity of labor (Weisdorf, 2005). At a larger scale of analysis — such as a band of foragers — such comparison is not relevant. In such a band, foragers adopt very different strategies. Some of them adopt very risky strategies, e.g., hunting large mammals which are quite rare, difficult to hunt and provide few calories compared to the energy spent during the hunt. Others prefer more secure strategies, such as gathering wild plants (e.g., nuts, pinions...) or animals (shellfish...) which are abundant, easy to collect and provide a very high ratio of calories. These differences in the economic behavior of foragers is however rational because those foragers belong to a same band in which there is a social rule, namely sharing food resources (and even beyond food resources). At least six different theories have been proposed to explain the existence and patterning of intra-group food sharing (Kaplan \& Gurven, 2005; Gurven \& Jaeggi, 2015); however the outcome of risk-reduction is consistent with all six. In other words, some foragers adopt risky strategies because they know that if their hunt fail, they will nevertheless have food provided by the other foragers of their band and through the sharing system. Therefore, a band of foragers cannot be viewed as a set of producers (or firms) competing on the market; through the sharing system, they behave in a cooperative manner and thus the behavior of any forager cannot be analyzed separately from the behavior of the band as a whole. An analogy between the market economy and the foraging economy can be established as follows. The foragers who belong to a same band have socio-economic relationships among them which are similar-even though they are less hierarchical - to the relationships between the employees of a given firm (Note 11). 


\subsection{Market Extension and the Transition from Cooperation to Competition}

We have previously recall that sharing - i.e., common sharing or communities sharing — was a central feature of foragers societies. In other words, cooperation among (unspecialized) foragers is the social norm. We now demonstrate that such norm cannot be maintained when foragers become specialized and involved in market transactions, i.e., when the transition under study is from case 1 to case 3, as presented in the previous section. Indeed, when foragers become specialists, any of them is no more facing the exploitation-exploration trade-off because, by definition, he is now specialized in the exploitation of a wild resource. Therefore, the division of labor between exploiters and explorers disappears, and the associated resources pooling system becomes unsustainable. Now the division of labor among foragers is not associated with sharing but with another "social norm", i.e., with the existence of the market. On the market the social norm between specialized foragers is now competition (it was cooperation between unspecialized foragers) and risk insurance is now provided by the "invisible hand" (it was initially provided by sharing). Thereby, we reach our first conclusion: when foragers get specialized in order to trade their surplus on the market, the relationship between them shifts from cooperation to competition. In other words, specialized foraging and trade are not consistent with what is considered as the natural socio-economic structure of foragers societies. Since the latter is deeply transformed, it is less likely that specialized foraging may lead to sustainable economic development. For instance a fundamental input in the foraging process is LEK and its transmission among foragers is closely associated with the norm of sharing. While LEK is clearly a public good, its production and transmission across foragers and generations of foragers becomes uncertain when sharing is removed by the market. Foraging is therefore unsustainable in the long term if it is specialized and market-oriented. The same conclusion has been reached when we have previously considered the evolution of foragers' economic profitability.

\section{From Mixed Economies and Self-Consumption to Specialization in Foraging or Farming and} Trade

In section 4 we have assumed that foraging was the only method available to get resources. Now we still consider a simplified economy but we enrich it: foraging still exists but farming is also possible. This implies that any people can be a forager and a farmer (this is case 2) at the same time (Note 12), i.e., that he has to decide how much effort (or working time) can be allocated to each activity in order to get resources (respectively extracted and produced resources). In addition to the previous decision relative to the distribution of his effort, any forager-farmer also has to decide either to self-consume his resources or systematically to trade his surplus and to rely on the market for his own consumption. The latter situation corresponds to case 4, defined as "foragers-farmers-traders".

In such economy, the main question seems to be under which circumstances people (foragers-farmers) decide to shift from self-consumption (case 2) to market-based consumption (case 4)? However, we have already answered to this question in the sections 4 and 5 of the present paper. Indeed, if people 
decide to only self-consume what they extract and produce, there is no exchange through the market and therefore their welfare will be at its lowest level. In other words, and once again according to Ricardo's theorem of comparative advantages, people will have welfare gain if they get specialized (in foraging or farming activities), derive a surplus and trade it on the market. Of course, and as seen previously, even if they get specialized, this does not mean that a sustainable economic development will be the long-term outcome associated with this decision. Thus, the important question is about what to do on the supply-side of the economy, i.e., to forage or to farm? In order to answer this question, we consider the various relationships that may exist between the outputs of both activities.

\subsection{Perfect Substitute Goods and Compared Productivities}

Such question has already been studied in the abundant literature about the transition from foraging to farming, i.e., as an explanation of the Neolithic revolution (Weisdorf, 2005). An implicit assumption in this literature is that the wild resources extracted and the farmed products are similar or very close substitutes (Note 13). Therefore, all these resources and products are exchanged on the same market; competition on the latter can be considered as perfect competition (when there is an infinite number of suppliers) or as Cournot (or quantity) competition (when the number of suppliers is low). The profitability of each activity depends on the supply-side conditions, i.e., on the respective costs of each activity. For simplicity, it is often assumed that the cost is only related to the labor input, and thus to the productivity of the latter. In other words, the decision to forage or to farm is directly defined by the comparison of the labor productivity in both activities. Two different outcomes are possible.

The first one is when the gap between labor productivities in both activities is small. In such situation, suppliers decide to forage and to farm, i.e., they do not become specialist. It is widely agreed that the labor productivity of foraging is low; thus if the gap with labor productivity in farming is low, it means that the latter productivity is also low. This may occur either during the early ages of agriculture (Smith, 2001) or in areas which are marginal (Note 14) for agriculture (Bellwood \& Oxenham, 2008, pp. 28-29). In both cases, labor productivity is low and therefore it is likely that foraging and farming do not provide any surplus and are only sufficient to ensure self-consumption. Without surplus, any economic development is hence impossible.

The second outcome is when the gap between labor productivities in both economic systems is large. This means that one activity is competitive while the other is not; after a while, the latter will disappear and only one activity (foraging or farming) will remain. This is the natural outcome of competition among suppliers of substitute goods on a same market. Let us assume that foraging is the more competitive, i.e., that labor productivity in foraging is strictly higher than in farming. The question is what will be the outcome in the long-term? In fact we have already answer to that question (see section 4) because this situation is when people decide to get specialized in foraging. As previously demonstrated, such situation is not sustainable in the long-term. 


\subsection{Different Goods, Dual Economy and Craft Industry}

The previous comparison between foraging and farming through the comparison of their respective productivity of labor is valid only if the outputs of both economic systems are close substitutes. However, we may consider many situations in which these outputs are different goods (Note 15) (i.e., are not substitute at all). Thus, if both economic systems lead to a surplus, each surplus is trade on a different market. Foragers and farmers are not (direct) competitors. The variation of the labor productivity in both activities is simply reflected by variation of the relative price of both goods.

In such situation, the economy is dual, with a sector of farmed products and a sector of foraged resources. Although both sectors are separated on the supply-side, they interact on the demand-side throughout the income distribution. From their activity, specialized foragers derive a surplus they trade, and hence they earn an income. They will use this income in order to buy resources and products in both market. Since the farming sector produces only edible products and the foraging sector provides non-edible wild resources (when outputs of both sectors are different goods), this means that foragers will tend to spend (most of) their income for buying farmed food products. Then, these foragers will tend to have no self-consumption, to be completely dependent on the market for all the edible products they consume (except the resources they forage). In other words, even if they still forage (the wild resources they trade) they tend to become very different from what is usually considered as "foragers". Indeed they constitute a subset of the agrarian economy which can be defined as an "industry". An economic development can emerge from a dual economy consisting of an agricultural sector and an industrial sector but its sustainability is dubious. Indeed, two situations can be associated with this industrial sector. In the first one, foragers are providing to the market wild resources that are not transformed, i.e., raw materials (e.g., bamboos). In exchange, they get from the market transformed products (e.g., flour, bread, biscuits) provided by the farming sector. While farmed and transformed products provided added value, foraged raw materials do not. Thus, the market exchange is likely to be characterize by a long-term decline in terms of trade. The latter means that, although they commercialize wild resources, foragers get impoverished with the passage of time, i.e., are not able to sustain an economic development. The second situation is when foragers extract and also transform wild resources before they sell them on the market. Because most foragers do not have access to large amounts of capital, they are not able to develop a "true" industry; hence, their activities are considered as a craft industry. In such a dual economy — with an agricultural sector and a craft industry- the role of foraging per se is minor. Indeed, most added-value of the craft industry is provided by craftsmen's skills rather than by the wild resources they use to produce crafts. In other words, it is not the foraging part of the craft process of production which sustain the economic development. 


\subsection{Substitutes and Differentiated Goods}

There also exist intermediary situations in which foraged resources and produced goods are neither similar (or perfect substitutes) nor different. This is when goods are substitutes and differentiated (Note 16). The goods perform the same basic functions but have differences in qualities such as type, style, quality, reputation, appearance, and location that tend to distinguish them from each other. To some extent this corresponds to what is also called "emerging middle ground" (Sills et al., 2011). The market structure is characterized by monopolistic competition which involves a great deal of non-price competition. Indeed, any forager as well as any farmer have a degree of control over price, i.e., he can raise his prices without losing all of his customers because any individual supplier's demand curve is downward sloping. For any people, the choice between foraging and farming leads to a specialization based on product differentiation.

The standard conclusion of monopolistic competition applied in our particular framework. This means that in the long run, a monopolistically competitive supplier will make zero economic profit. In other words, foraging and farming co-exist, even in the long run, but they may not lead to an accumulation process consistent with sustainable economic development.

\subsection{Ecological Inheritance and Externality}

Until now, we have assumed - at least implicitly — that foraging and farming were two independent economic activities between which people have to distribute their effort. However, these two activities can be dependent, beyond the willing of people. In other words there may exist some externalities linking foraging and farming. Such externalities exist because the productivities of foraging as well as of farming depend on the ecosystem and that both economic systems also have an influence on the ecosystem. In HBE, the latter is labeled the effects of ecological inheritance (Freeman et al., 2015). For instance, agriculture (e.g., fields of crop) attracts game and thus provides, in the short-term, a positive externality for hunters. However such externality is likely to be negative in the long-term because agriculture modifies the landscape, implies deforestation, i.e., leads to an ecosystem which is less favorable for wild animals, and thus for hunters. Because such externalities can be positive at one stage and negative later, when they are taken into account in the analysis it is difficult to reach any definitive conclusion about what is the most efficient decision, foraging or farming.

\section{Conclusion}

We have previously demonstrated that intensive foraging and commercialization of wild resources cannot lead to a sustainable economic development as it was thought when the MDGs were adopted. The main reason explaining such result is that while the extraction of wild resources is a technique which can be compared to the production of goods, foraging cannot be restricted to a technique and even to an economic system. Indeed, foraging is a socio-economic system, i.e., an economic system which is socially embedded (Note 17). In a foraging economy-whatever the period it exists-social embeddedness is ubiquitous because sharing is the main feature of such economy (Lee, 2004; Lee \& 
Daly, 2004). From an economic point of view, the supply and the demand of any foraging economy - even though such economy is very small, e.g., associated only with a band of foragers - are not independent (Note 18). Thus, any change to the supply-side has consequences on the demand-side, and vice et versa.

Thus, the shift from a foraging economy dedicated to self-consumption to a specialized and intensive foraging economy devoted to trade wild resources on the market is likely to have two negative outcomes associated with the disappearance of sharing. First, many foragers may suffer of the shift from a cooperative to a competitive system, i.e., many of them can be impoverished (especially those who adopt risky strategies and/or are unlucky). Second, and even though it was not the aim of the present paper (Note 19), we may surmise that many wild resources can be endangered due to excessive extraction. Both outcomes are simply opposite to what was the initial wish of those who fostered forest peoples to exploit and commercialize forest products.

\subsection{Market Extension and Its Socio-Economic Consequences on Sharing}

However, the link between specialized foraging and commercialization, and the breakdown of communal sharing must be qualified because the latter seems more related to market extension. As shown previously, it is true that the existence of the market allows increased specialization and greater division of labor. It may result in a breakdown in communal rules. However, it is not clear that this is due to specialization per se or to division of labor. Indeed, the argument that increasing division of labor results in a lack of co-operation and ultimately unsustainable economic development must be qualified. Division of labor occurred in most primitive societies. Australian Aboriginal women mostly gathered food whereas the men hunted large animals and made important tools. Sharing of food was very important for their economic security. Similarly, it is not systematic that increased specialization results in foragers no longer co-operating. It could be more vital to do so because of the risks associated with specialization.

However, the effects of markets are not only to increase the division of labor and specialization. Indeed, it is probably more the effects of market extension, rather than the increased division of labor and specialization, which results in a breakdown in communal sharing. Such extension has economic as well as social consequences on foragers. Concerning the former, when they trade on the market the wild resources they harvest, foragers thereby have access to a cash economy. This could help them to reduce their incentive for co-operation because money can be used as a store of values and savings can be drawn on in time of need. This is not to say that villagers did not have other stores of value in some cases prior to cash. For example, livestock where sometimes used as a store of value, and still are in some cases. However, because money or cash income is intrinsically associated with perfect liquidity, it tends to be preferred to any other store of value. Concerning the latter, i.e., the social consequence of market extension, one should noticed that the universe of tribals expands as they come into contact with the market system of non-tribals. This changes their outlook and possibilities, and places new pressures on them, e.g., to send their children to school. 


\subsection{Sharing, Foraging and Biodiversity Conservation}

The existence of sharing has also consequences on biodiversity conservation. When it is compared to a production process, the extraction of wild resources is characterized as different for two reasons. On the one hand, it is associated with diminishing marginal returns because, mechanically any extraction reduces the remaining stock of wild resources and therefore requires additional effort for the subsequent extraction. On the other hand, excessive extraction-beyond the carrying capacity—can lead to depletion or extinction of the wild resources. Both explanations are valid if we consider a microeconomic analysis, i.e., the impact on the nature of a particular forager's actions. However these explanations must be qualified when are analyzed the impacts of a band of foragers' actions. Indeed, once again sharing is present among foragers belonging to a same band. Moreover, the sharing rules can be interpreted as an implicit tax on the harvest of renewable resources (Chakraborty, 2007). Such tax lowers the marginal return to resource harvesting, which reduces effort and increases the stock of wild resources. In other words, foraging does not necessarily lead to overexploitation of wild resources. On the contrary, in a foraging economy, sharing avoids waste of food and favors resource conservation because it reduces foragers' incentives to extract wild resources.

Of course, the previous conclusion depends on the land tenure system. If there are no communal rules, i.e., if there is a de facto open-access system, then items of greatest use value would be threatened with annihilation whether marketed or not. However, the threat would be greater if they could be marketed because this would give scope to buy commodities having considerable utility. When suitable communal rules exist, foragers may adopt practices which favor their desired species, thereby altering biodiversity. The fire-stick culture of Australian Aborigines is reputed to have done this, even though these people engaged in sharing to some extent. Given communal rules, what was conserved would have been influenced by use value and presumably was a common incentive to conserve items of greatest value. This did not necessarily conserve biodiversity.

\section{Acknowledgement}

The Author wishes to thank Professor Clem Tisdell for his constructive comments on an early version of the paper. The usual caveat applies.

\section{References}

Ahenkan, A., \& Boon, E. (2011). Non-Timber Forest Products (NTFPs): Clearing the Confusion in Semantics. Journal of Human Ecology, 33(1), 1-9.

Bellwood, P., \& Oxenham, M. (2008). The expansions of farming societies and the role of the Neolithic demographic transition. In J.-P. Bocquet-Appel, \& O. Bar-Yosef (Eds.), The Neolithic Demographic Transition and its Consequences (pp. 13-34). Dordrecht: Springer.

Berger-Tal, O. N., Meron, J., \& Saltz, D. E. (2014). The Exploration-Exploitation Dilemma: A Multidisciplinary Framework.

ONE, $\quad 9(4)$, e95693. 
http://dx.doi.org/10.1371/journal.pone.0095693

Bharucha, Z., \& Pretty, J. (2010). The roles and values of wild foods in agricultural systems. Phil. Trans. R. Soc. B, 365, 2913-2926. http://dx.doi.org/10.1098/rstb.2010.0123

Chakraborty, R. N. (2007). Sharing culture and resource conservation in hunter-gatherer societies. Oxford economic papers, 59(1), 63-88. http://dx.doi.org/10.1093/oep/gpl025

Chambers, R. J. (1987). Sustainable Livelihoods Environmental Development: Putting Poor People First (Working Paper). Institute of Development Studies, University of Sussex, Brighton.

Charnov, E. L. (1976). Optimal foraging, the marginal value theorem. Theor. Pop. Biol., 9, 129-136.

Childe, V. G. (1936). Man Makes Himself. London: Watts.

Cohen, J. D., McClure, S. M., \& Yu, A. J. (2007). Should I stay or should I go? How the human brain manages the trade-off between exploitation and exploration. Phil Trans $R$ Soc B, 362, 933-942.

Cooper, A. et al. (2015). Abrupt warming events drove late Pleistocene Holarctic megafaunal turnover. Science, 349, 602-606.

Delang, C. O. (2006a). The role of wild food plants in poverty alleviation and biodiversity conservation in tropical countries. Progress in Development Studies, 6(4), 275-286.

Delang, C. O. (2006b). Not just minor forest products: The economic rationale for the consumption of wild food plants by subsistence farmers. Ecological Economics, 59, 64-73. http://dx.doi.org/10.1016/j.ecolecon.2005.10.006

Delton, A. W., \& Robertson, T. E. (2012). The social cognition of social foraging: Partner selection by underlying valuation. Evolution and human behavior, 33, 715-725.

Freeman, J., Peeples, M., \& Anderies, J. M. (2015). Toward a theory of non-linear transitions from foraging to farming. Journal of Anthropological Archaeology, 40, 109-122. http://dx.doi.org/10.1016/j.jaa.2015.07.001

Giraldeau, L.-A., \& Caraco, T. (2000). Social foraging theory. Princeton (NJ): Princeton University Press.

Gurven, M., \& Jaeggi, A. V. (2015). Food sharing. In R. Scott, \& S. Kosslyn (Eds.), Emerging Trends in the Social and Behavioral Sciences (pp. 1-12). John Wiley \& Sons.

Kaplan, H., \& Gurven, M. (2005). The natural history of human food sharing and cooperation: A review and a new multi-individual approach to the negotiation of norms. In H. Gintis, S. Bowles, R. Boyd, \& E. Fehr (Eds.), Moral sentiments and material interests: The foundations of cooperation in economic life. Cambridge, MA: MIT Press.

Keeley, L. H. (1988). Hunter-Gatherer Economic Complexity and "Population Pressure": A Cross-Cultural Analysis. Journal of Anthropological Archaeology, 7, 373-411.

Kelly, R. L. (1995). The Foraging Spectrum: Diversity in Hunter-Gatherer Lifeways. Washington and London: Smithsonian Institution Press.

Lee, R. B. (2004). Power and property in twenty-first century foragers: A critical examination. In T. Widlok, \& T. Wolde (Eds.), Power and equality: Encapsulation, commercialization, 
discrimination (pp. 16-31). Oxford: Berg Publishing.

Lee, R. B., \& Daly, R. H. (Eds.). (2004). Introduction: Foragers and others. The Cambridge Encyclopaedia of Hunters and Gatherers (pp. 1-19). New Delhi: Cambridge University Press.

McLain, R. J., Hurley, P. T., Emery, M. R., \& Poe, M. R. (2014). Gathering "wild” food in the city: Rethinking the role of foraging in urban ecosystem planning and management. Local Environment, $19(2), 220-240$

Mehlorn, K. et al. (2015). Unpacking the exploration-exploitation trade-off: A synthesis of human and animal literatures. Decision, 2(3), 191-215.

Morgan, L. H. (1877). Ancient Society, or Researchers in the Line of Human Progress from Savagery, through Barbarism to Civilization. London: Macmillan and Co.

Morrison, K. D. (2005). Historicizing foraging in Asia: Power, history, and ecology of Holocene hunting and gathering. In M. Stark (Ed.), An Archaeology of Asia (pp. 279-302). Basil Blackwell, New York.

North, D. C., \& Thomas, R. P. (1977). The first economic revolution. The Economic History Review, Second Series, 30, 229-241.

Peters, C. M., Gentry, A. H., \& Mendelsohn, R. O. (1989). Valuation of an Amazonian forest. Nature, $339,655-656$

Polanyi, K. (1944). The great transformation. New York: Holt, Rinehart.

Price, T. D., \& Brown, J. (1985). Prehistoric Hunter-Gatherers: The Emergence of Cultural Complexity. San Diego, Calif: Academic Press.

Pryor, F. L. (2004). From Foraging to Farming: The So-called “Neolithic Revolution”. In A. J. Field, G. Clark, \& W. A. Sundstrom (Eds.), Research in Economic History (pp. 1-41). Boston, MA: Elsevier/JAI.

Pyke, G. H., Pulliam, H. R., \& Charnov, E. L. (1977). Optimal foraging: A selective review of theory and tests. Quarterly Review of Biology, 52, 137-154. http://dx.doi.org/10.1086/409852

Renfrew, C., \& Bahn, P. (2012). Archaeology. Theories, Methods and Practice (6th ed.). Thames \& Hudson Ltd: London.

Ricardo, D. (1817). On the Principles of Political Economy and Taxation. John Murray, London. Retrieved from http://www.econlib.org/library/Ricardo/ricP.html

Ruiz-Pérez, M. et al. (2004). Markets drive the specialization strategies of forest peoples. Ecology and Society, 9(2), 4. Retrieved from http://www.ecologyandsociety.org/vol9/iss2/art4

Saavreda, S. et al. (2013). Foraging under conditions of short-term exploitative competition: The case of stock traders. Proceedings of the Royal Society B. http://dx.doi.org/10.1098/rspb.2012.2901

Sassaman, K. E. (2004). Complex hunter-gatherers in evolution and history: A North American perspective. Journal of Archaelogical Research, 12(3), 227-280.

Service, E. R. (1975). Origins of the State and Civilization: The Process of Cultural Evolution. W. W. Norton \& Company, Inc. New York. 
Shackleton, S. et al. (2008). Links between the Local Trade in Natural Products, Livelihoods and Poverty Alleviation in a Semi-arid Region of South Africa. World Development. http://dx.doi.org/10.1016/j.worlddev.2007.03.003

Sills, E. et al. (2011). Evolving Perspectives on Non-timber Forest Products. In S. Shackleton et al. (Eds.), Non-Timber Forest Products in the Global Context. Tropical Forestry 7, Springer-Verlag: Berlin Heidelberg. http://dx.doi.org/10.1007/978-3-642-17983-9_2

Smith, A. (2008). In K. Sutherland (Ed.), An Inquiry into the Nature and Causes of the Wealth of Nations: A Selected Edition. Oxford Paperbacks, Oxford, UK.

Smith, B. (2001). Low-level food production. Journal of Archaeological Research, 9, 1-43.

Smith, V. L. (1975). The primitive hunter culture, Pleistocene extinction, and the rise of agriculture. Journal of Political Economy, 83, 727-755.

Stanley, D. et al. (2012). Is Non-Timber Forest Product Harvest Sustainable in the Less Developed World? A Systematic Review of the Recent Economic and Ecological Literature. Ethnobiology and Conservation, $1,9$.

Stiles, D. (1994). Tribals and trade: A strategy for cultural and ecological survival. AMBIO. A journal of the human environment, 23(2), 106-111.

Stiles, D. (1998). The Mikea hunter-gatherers of southwest Madagascar: Ecology and socioeconomics. African Study Monographs, 19(3), 127-148.

Stiles, D. (2001). Hunter-gatherer studies: The importance of context. African Study Monographs, 26, 41-65.

Svizzero, S. (2016). Foraging Wild Resources: Evolving Goals of an Ubiquitous Human Behavior. Anthropology, 4(161). http://dx.doi.org/10.4172/2332-0915.1000161

Svizzero, S., \& Tisdell, C. (2014). Theories about the Commencement of Agriculture in Prehistoric Societies: A Critical Evaluation. Rivista di Storia Economica, 3, 255-280. http://dx.doi.org/10.1410/78237

Svizzero, S., \& Tisdell, C. (2015). The Persistence of Hunting and Gathering Economies. Social Evolution and History, 14(2), 3-25.

Svizzero, S., \& Tisdell, C. (2016). The Post-2015 Development Agenda: A Critical Analysis. Journal of Self-Governance and Management Economics, 4(1), 72-94.

Swanson, T. (1994). The international regulation of extinction. Washington Square, NY: New York University Press.

Tisdell, C. A. (2014). Human values and biodiversity conservation: The survival of wild species. Cheltenham, and Northampton, MA: Edward Elgar.

Tisdell, C. A. (2015). Sustaining Biodiversity and Ecosystem Functions: Economic Issues. Cheltenham, and Northampton, MA: Edward Elgar.

Weisdorf, J. L. (2005). From foraging to farming: Explaining the Neolithic Revolution. Journal of Economic Surveys, 19, 561-586. 
Winterhalder, B., \& Kennett, D. J. (Eds.). (2006). Behavioral ecology and the transition from hunting and gathering to agriculture. Behavioral Ecology and the Transition to Agriculture (pp. 1-21). Berkeley: University of California Press.

\section{Notes}

Note 1. See Svizzero and Tisdell (2016) for the difficulties encountered in order to tackle simultaneously MDGs and Sustainable Development Goals.

Note 2. For a recent and detailed analysis of the definition(s) of NTFPs, see Ahenkan and Boon (2011).

Note 3. See Tisdell (2014) for a complete study of this topic, and especially chapter 16.

Note 4. It remains contentious whether or not feral animals should be considered as wild, even though some of them are hunted.

Note 5. As some modern hunters or (no kill) fishers do; see also wild plants gathered for gardening, wild animals caught in the wild and kept in zoo, aquarium or as pets (...).

Note 6. Ricardo's theorem also includes a condition on the level of the international relative price of exchanged goods in order to ensure the existence of mutual welfare gains; here we do not consider it because it is beyond the scope of this paper.

Note 7. These 61 cases of the commercial production and trade of (different) non-timber forest products from Asia, Africa, and Latin America — encompass different strategies adopted by forest peoples such as the four cases we have defined but also some cases of forest peoples who do not forage.

Note 8. Even though the Pleistocene overkill hypothesis has been recently discredited and replaced by explanations based on the influences of climate change (Cooper et al., 2015).

Note 9. E.g., the Natufians in the Levant, the Ertebolle culture in Northern Europe, the Pitted Ware Culture in Scandinavia, the Jomon culture in Japan (...), all existed during the prehistoric period. More recent examples are provided by peoples who were living (until at least $1500 \mathrm{AD}$ ) on the American North-West coast.

Note 10. E.g., wild cereals, anadromous fish, shellfish, sea mammals (...).

Note 11 . As pointed out by what economists call the "transaction costs approach".

Note 12. Otherwise we may also assume that the total population is divided in two separate groups, foragers and farmers, and that the relative weight of both groups is evolving over time.

Note 13. E.g., bushmeat (provided by hunting game) and meat of reared animals are very close substitutes. Cereals, harvested from the wild or cultivated, can also be considered as close substitutes.

Note 14. E.g., due to harsh climatic conditions or unfertile soils.

Note 15. E.g., when farmers produce any edible plants (crop) and that foragers extract non edible wild resources used as raw materials (bamboos, rattan, medicinal plants...).

Note 16. Goods can also be described as close but imperfect substitutes.

Note 17. The Social embeddedness position is associated with the "substantivist school" in anthropology, identified especially with Polanyi (1944). 
Note 18. One may even say that there is an analogy between sharing and macroeconomic analysis. Indeed, according to Keynes, there is in the latter a link (at the macroeconomic level) between the demand and the supply through the income distribution. In the typical foraging economy, such link also exists. The wild resources that are shared are considered by those who forage as an implicit tax, and as a subsidy by those who receive these resources. Sharing then affects the whole income distribution, exactly as the Welfare State did in the Keynesian macroeconomic analysis.

Note 19. For more on this topic, see Tisdell $(2014,2015)$. 